\title{
Multifractal correlations of the local density of states in dirty superconducting films
}

\author{
M. Stosiek, ${ }^{1}$ F. Evers, ${ }^{2}$ and I. S. Burmistrov ${ }^{3,4}$ \\ ${ }^{1}$ Physics Division, Sophia University, Chiyoda-ku, Tokyo 102-8554, Japan \\ ${ }^{2}$ Institute of Theoretical Physics, University of Regensburg, D-93040 Regensburg, Germany \\ ${ }^{3}$ L.D. Landau Institute for Theoretical Physics, Akademika Semenova av. 1-a, 142432 Chernogolovka, Russia \\ ${ }^{4}$ Laboratory for Condensed Matter Physics, HSE University, 101000 Moscow, Russia
}

(Received 19 July 2021; accepted 27 September 2021; published 4 November 2021)

\begin{abstract}
Mesoscopic fluctuations of the local density of states encode multifractal correlations in disordered electron systems. We study fluctuations of the local density of states in a superconducting state of weakly disordered films. We perform numerical computations in the framework of the disordered attractive Hubbard model on two-dimensional square lattices. Our numerical results are explained by an analytical theory. The numerical data and the theory together form a coherent picture of multifractal correlations of local density of states in weakly disordered superconducting films.
\end{abstract}

DOI: 10.1103/PhysRevResearch.3.L042016

Superconductivity and Anderson localization are two physical effects that result in a rich variety of quantum phenomena. Initially, it was believed that nonmagnetic disorder does not affect superconductivity (so-called "Anderson theorem") [1-3]. Later it became clear that Anderson localization in the presence of disorder cannot only suppress superconductivity [4-7], but also lead to superconductor-to-insulator transition [8] (see Refs. [9-11] for a review). In the presence of Coulomb interaction even a weak disorder was predicted to be sufficient to destroy a superconducting state [12-21].

In the case of a short-ranged electron-electron interaction (e.g., if Coulomb interaction is screened), Anderson localization can enhance the superconducting transition temperature, $T_{c}$ [22-25]. This surprising phenomenon originates from the multifractality of electron wave functions in disordered media [26]. The latter leads to an increase in the effective attraction and, consequently, to growth of $T_{c}$. Recently, theoretical predictions of Refs. [22-25] have been corroborated by numerical solutions of the disordered attractive Hubbard model $[27,28]$ and experimental observations of enhancement of $T_{c}$ in disordered niobium dichalcogenide monolayers $[29,30]$. The multifractally enhanced superconductivity is predicted to be accompanied by strong mesoscopic fluctuations of the local order parameter and the local density of states (LDOS) [23,28,31-35].

Point-to-point fluctuations of the tunneling LDOS, frequently termed as an emergent electronic granularity, have been observed experimentally in many studies of disordered superconducting films [29,30,36-42]. The measurements yield LDOS maps that are used to extract the statistics of the energy gap and the LDOS maximum. Recently, the spatial

Published by the American Physical Society under the terms of the Creative Commons Attribution 4.0 International license. Further distribution of this work must maintain attribution to the author(s) and the published article's title, journal citation, and DOI. correlations of the energy gaps have been analyzed, and the emergence of a well-defined spatial scale has been demonstrated [41].

Multifractally enhanced superconductivity was predicted to occur in a relatively narrow region in the disorderinteraction plane [24,25]. However, significant point-to-point fluctuations of the LDOS have been reported in many experiments on superconducting films that do not demonstrate enhancement of $T_{c}$ with disorder. Therefore, there is a question about the origin of an emergent electronic granularity, especially in weakly disordered superconducting films.

In this Letter we investigate numerically and with analytical means, the fluctuations of the LDOS in the superconducting state of weakly disordered films. We perform numerical computations of disordered attractive Hubbard model on a two-dimensional square lattice. We focus on the energy dependence of the variance of the LDOS. Even at the very low level of disorder we observe pronounced fluctuations of the LDOS. Our numerical findings are complemented with the analytical theory for the fluctuations of the LDOS. The most striking observation both in the numerics and in the theory is the logarithmic divergence of the LDOS variance (at energies higher than the energy gap) with a system size $L$. Such divergence is a direct signature of multifractal behavior of the LDOS, originating from mesoscopic fluctuations. We demonstrate that the numerical findings and the analytical results form a coherent picture of multifractal correlations of the LDOS in weakly disordered superconducting films.

Numerics. We consider the attractive- $U$ Hubbard model [43] on the square lattice in two dimensions with double-periodic boundary conditions. Within the mean-field approximation the Hamiltonian reads $(U>0)$

$$
\begin{aligned}
\hat{H}= & -t \sum_{\langle i, j\rangle, \sigma} \hat{c}_{i, \sigma}^{\dagger} \hat{c}_{j, \sigma}+\sum_{i, \sigma}\left[V_{i}-\mu-U n\left(\boldsymbol{r}_{i}\right) / 2\right] \hat{n}_{i, \sigma} \\
& +\sum_{i} \Delta\left(\boldsymbol{r}_{i}\right) \hat{c}_{i, \uparrow} \hat{c}_{i, \downarrow}+\text { H.c. }
\end{aligned}
$$



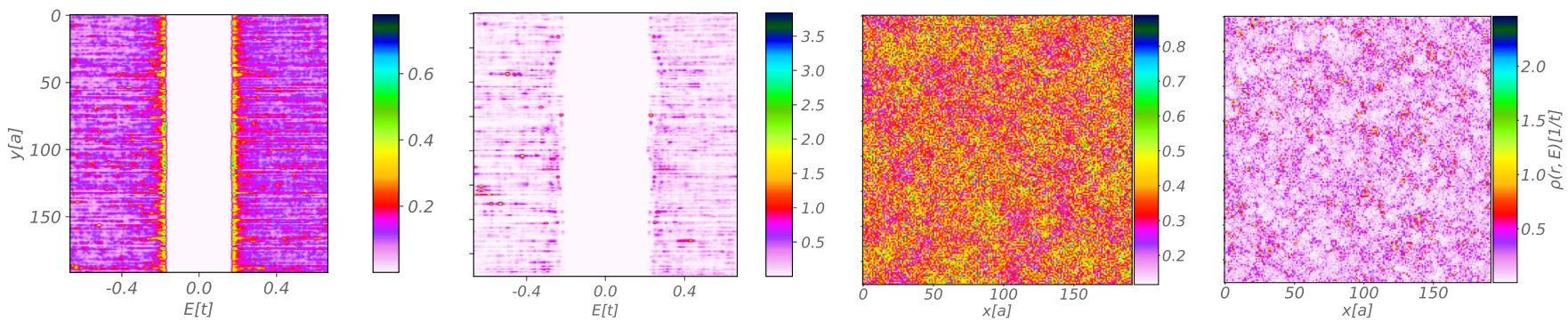

FIG. 1. Left panels: LDOS-line cuts of typical samples along the axial $y$ direction of the lattice and $x=0$ for $W=0.5$ (outer left panel) and $W=1.25$ (middle left). Right panels: LDOS maps of the same samples as in the left panels for $W=0.5$ (middle right) and $W=1.25$ (outer right) on the entire lattice for $E=E_{\max }$ with the peak energy of the disorder-averaged DoS $E_{\max }$. (Parameters: zero temperature, 0.3 filling, $U=2.2 t, L=192$, and $N_{\mathcal{C}}=8192$.)

Here $\hat{c}_{j, \sigma}^{\dagger}$ and $\hat{c}_{j, \sigma}$ stand for the creation and annihilation operators of a fermion with spin projection $\sigma= \pm 1 / 2$ at a site $j$. An on-site random potential is drawn from the box distribution $V_{i} \in[-W, W]$. The chemical potential $\mu$ fixes the filling factor to 0.3 ; throughout this Letter the interaction is taken as $U=2.2 t$. The local occupation number $n\left(\mathbf{r}_{i}\right)$ and the pairing amplitude $\Delta\left(\mathbf{r}_{i}\right)$ are determined self-consistently,

$$
n\left(\boldsymbol{r}_{i}\right)=\sum_{\sigma}\left\langle\hat{n}_{i, \sigma}\right\rangle, \quad \Delta\left(\boldsymbol{r}_{i}\right)=U\left\langle\hat{c}_{i, \downarrow}^{\dagger} \hat{c}_{i, \uparrow}^{\dagger}\right\rangle,
$$

where $\hat{n}_{i, \sigma}=\hat{c}_{i, \sigma}^{\dagger} \hat{c}_{i, \sigma}$ and the average is taken with respect to the equilibrium density matrix corresponding to the Hamiltonian (1) at zero temperature. We solve Eqs. (1) and (2) iteratively and terminate the self-consistency cycle [44] when $\alpha^{(n)}$, the relative change in $\Delta\left(\boldsymbol{r}_{i}\right)$ in iteration $n$, is at each site $\boldsymbol{r}_{i}$ smaller than some $\alpha$,

$$
\alpha^{(n)}=\max _{\boldsymbol{r}_{i}}\left|\frac{\Delta^{(n)}\left(\boldsymbol{r}_{i}\right)-\Delta^{(n-1)}\left(\boldsymbol{r}_{i}\right)}{\Delta^{(n-1)}\left(\boldsymbol{r}_{i}\right)}\right|<\alpha .
$$

For system size $L=192$, we chose $\alpha=10^{-3}$, and for the smaller system sizes, we chose $\alpha=10^{-4}$. We employ the kernel polynomial method [46] to compute $n\left(\boldsymbol{r}_{i}\right), \Delta\left(\boldsymbol{r}_{i}\right)$, and the LDOS. The underlying expansion of the time-evolution operator in Chebyshev polynomials to order $N_{\mathcal{C}}$ causes Gibbs oscillations that we deal with employing the Jackson kernel, see Ref. [34] for further computational details. The ensemble averaging over observables involves, typically, several hundred samples.

The dependence of the LDOS on energy $E$ and the spatial coordinate $y$ along a cut through the sample is shown in Fig. 1 for two values of disorder $W$. At weak disorder $W=0.5$ (left panel in Fig. 1) fluctuations of the local gap are almost absent and only after an increase up to $W=1.25$, the local gap fluctuations become more visible albeit being still small (middle panel in Fig. 1).

This observation is qualitatively consistent with an analytical result for the relative fluctuations of the local order parameter $\left\langle[\delta \Delta(\boldsymbol{r})]^{2}\right\rangle /\langle\Delta(\boldsymbol{r})\rangle^{2}=[4 /(\pi g)] \ln \left(\xi_{0} / \ell\right)[35]$, where $g$ represents the dimensionless conductance in the normal state, $\xi_{0}$ represents the coherence length at zero temperature, and $\ell$ represents the mean free path. At weak disorder, i.e., large $g$, fluctuations of the local order parameter are small. With an increase in disorder, i.e., decreasing $g$, the fluctuations of the local order parameter becomes more pronounced.
The dependence of the average LDOS and its variance on $E$ are presented in Fig. 2 for two values of disorder. With an increase in disorder the maximum in average LDOS becomes less pronounced as expected. The energy dependence of the LDOS variance has a form similar to the average LDOS, in particular, the variance has the maximum. We note that this maximum is situated at energy $\tilde{E}_{\max }$ which is smaller than $E_{\max }$. With an increase in disorder the LDOS variance increases.

Theory. In order to understand salient features of the numerical data, we consider two-dimensional fermions with a BSC-type attraction in the presence of a white-noise random potential, i.e., the continuum limit of the Hamiltonian (1) without the Hartree term. In the regime of a weak disorder, one can neglect the spatial dependence of the order parameter $\Delta$. Then the LDOS at a given realization of disorder can be written as

$$
\rho(E, \boldsymbol{r})=\sum_{a ; s= \pm} \varphi_{a}^{2}(\boldsymbol{r})\left(1+\varepsilon_{a} / E\right) \delta\left(E-s \sqrt{\varepsilon_{a}^{2}+\Delta^{2}}\right)
$$

where $\varepsilon_{a}$ and $\varphi_{\alpha}(\boldsymbol{r})$ are eigenenergies and eigenfunctions of the single-particle Hamiltonian in the absence of $\Delta$. Using the well-known results for statistics of eigenenergies and eigenfunctions of a weakly disordered noninteracting Hamiltonian [47], we compute the mean and variance of the local density of states from Eq. (4).

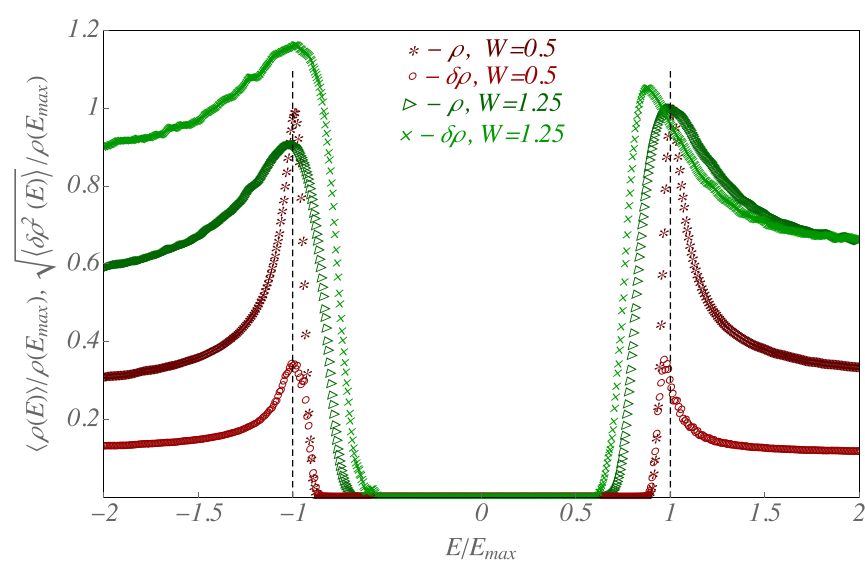

FIG. 2. The numerical data for the average LDOS and the LDOS variance for $W=0.5$ and $W=1.25$. 
The disorder-average density of states is given as $\langle\rho(E)\rangle=\rho_{0} \operatorname{Re} X_{E}$, where $X_{E}=E / \sqrt{E^{2}-\Delta_{E}^{2}}$. Here $\Delta_{E}$ is the energy-dependent gap function. We emphasize that such an energy dependence appears naturally in a more accurate treatment of the disordered electrons in the presence of attraction [35]. Although the energy dependence of $\Delta_{E}$ can be derived microscopically [35] for a sake of simplicity, we will use the phenomenological Dynes ansatz $\Delta_{E}=\Delta E /(E+i \Gamma)$ with $\Gamma \ll \Delta$ [48]. We note that the maximum of the average DOS is on the order of $\rho_{0} \sqrt{\Delta / \Gamma}$ and is situated at $E_{\max } \simeq \Delta+\Gamma / \sqrt{3}$. It is natural to expect that $\Gamma$ is enhanced by increasing disorder, so that the peak value reduces and the peak position shifts to larger values. Our numerical data, Fig. 2 are in qualitative agreement with this.

In order to compute the LDOS variance, we use the following well-known result for the irreducible part of the dynamical structure factor of noninteracting electrons in the diffusive regime (see, e.g., Ref. [49]),

$$
\begin{gathered}
\sum_{a, b}\left\langle\varphi_{a}^{2}(\boldsymbol{r}) \delta\left(E-\varepsilon_{a}\right) \varphi_{b}^{2}(\boldsymbol{r}) \delta\left(E^{\prime}-\varepsilon_{b}\right)\right\rangle_{\mathrm{irr}} \\
\simeq \frac{\rho_{0}}{2 \pi} \int \frac{d^{2} \boldsymbol{q}}{(2 \pi)^{2}} \frac{D q^{2}}{\left(D q^{2}\right)^{2}+\left(E-E^{\prime}\right)^{2}} .
\end{gathered}
$$

Here $D=g /\left(4 \pi \rho_{0}\right)$ denotes the diffusion coefficient. Using Eqs. (4) and (5), we find the variance of the normalized local DOS at $T=0$ to the lowest order in $1 / g$,

$$
\begin{aligned}
\sigma^{2} \equiv & \frac{\left\langle[\delta \rho(E, \boldsymbol{r})]^{2}\right\rangle}{\langle\rho(E)\rangle^{2}} \\
= & \frac{4}{\pi g} \operatorname{Re}\left[\ln \frac{L_{E}}{\ell}-\frac{1+\left|X_{E}\right|^{2}}{4\left(\operatorname{Re} X_{E}\right)^{2}} \ln \left(\frac{E_{\mathrm{Th}}}{E}+\operatorname{Im} \frac{1}{X_{E}}\right)\right. \\
& \left.+\frac{1-X_{E}^{2}}{4\left(\operatorname{Re} X_{E}\right)^{2}} \ln \left(\frac{E_{\mathrm{Th}}}{E}-\frac{i}{X_{E}}\right)\right] .
\end{aligned}
$$

Here $E_{\mathrm{Th}}=D /\left(2 L^{2}\right)$ stands for the Thouless energy and $L_{E}=\sqrt{D /(2 E)}$. Also we extend the result for the variance to the energy-dependent gap function(see Ref. [35] for details). Below we assume that the following condition holds $\Delta \gg \sqrt{\Gamma \Delta} \gg E_{\mathrm{Th}} \gg \Gamma$. This corresponds to the parameters of our numerical analysis.

Specifically, Eq. (6) predicts that at energies outside the gap-region $(E-\Delta) \gtrsim \Delta \gg \Gamma$, the normalized variance becomes almost independent of $E$, and, in particular, is logarithmically divergent with the system size,

$$
\left\langle[\delta \rho(E, \boldsymbol{r})]^{2}\right\rangle /\langle\rho(E)\rangle^{2} \simeq[4 /(\pi g)] \ln (L / \ell) .
$$

The prefactor in front of $\ln L$ in Eq. (7) coincides with the known result for the multifractal exponent for a weakly disordered metal $\Delta_{2}=-4 /(\pi g)$ [26]. The result (7) implies that the spatial correlation function of the LDOS at $(E-\Delta) \gtrsim \Delta \gg \Gamma$ behaves as $\left\langle\left[\rho(E, \boldsymbol{r}) \rho\left(E, \boldsymbol{r}^{\prime}\right)\right\rangle \propto\left(L /\left|\boldsymbol{r}-\boldsymbol{r}^{\prime}\right|\right)^{-\Delta_{2}}\right.$. Such powerlaw behavior is surprising for the system with the spectral gap and the finite coherence length $\xi_{0}$.

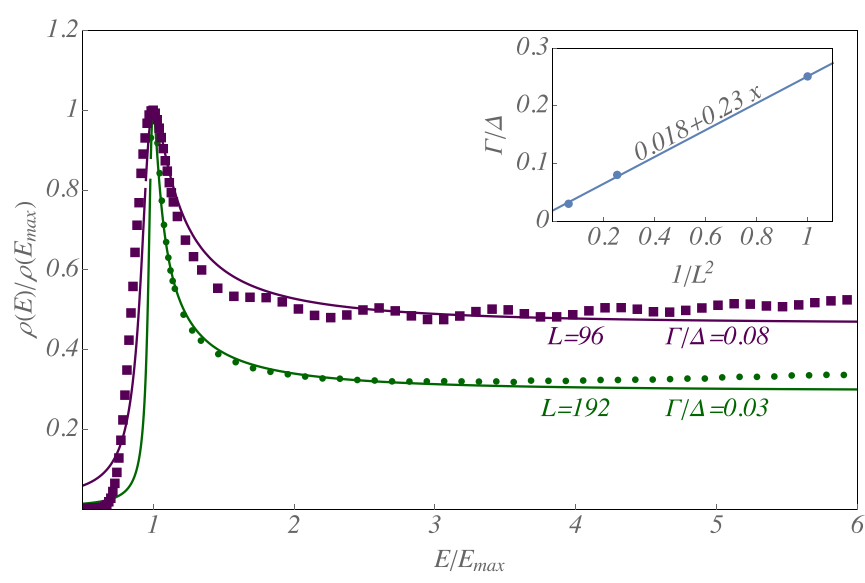

FIG. 3. The average DOS. Comparison between the numerics and the Dynes expression. The position of the maximum is $E_{\max } \approx$ 1 for $L=192$ and $E_{\max } \approx 1.08$ for $L=96$. [Parameters: $W=$ $0.5 t, U=2.2 t$, and $N_{\mathcal{C}}=2048(L=96), 8192(L=192)$.] The inset: Dynes parameter $\Gamma$ versus system size for $L=48,96,192$.

This logarithmic characteristics is a manifestation of multifractality in the superconducting state. It can be understood on the basis of Eq. (4). To obtain the variance, each side of (4) needs to be squared; two different contributions to the LDOS-variance arise: The first one correlates the electronlike part of the LDOS $[s=+$ in Eq. (4)] with the holelike part $(s=-)$. This contribution is sensitive to the gap since the energy difference between electron and holelike states cannot fall below $2 \Delta$. The second contribution resembles correlations between purely electronlike or holelike states. The corresponding energy difference can be arbitrarily small even in the presence of the gap and, therefore, fourth-order moments of wave-function amplitudes can exhibit significant correlations that are the characteristic precursors of multifractality.

Multifractality of the LDOS can be seen in the average values of the higher moments of $\rho(E, \boldsymbol{r})$. In the regime of weak disorder $g \gg 1$, the scaling of higher moments of the LDOS is fully determined by the second moment $\left\langle\rho^{q}(E, \boldsymbol{r})\right\rangle=\langle\rho(E)\rangle^{q}\left[\left\langle\rho^{2}(E, \boldsymbol{r})\right\rangle /\langle\rho(E)\rangle^{2}\right]^{q(q-1) / 2} \quad[32,35,50]$. Therefore, using the result (6) we can find the distribution function for the LDOS. After introducing the logarithm of the normalized LDOS, $x=\ln [\rho(E, \boldsymbol{r}) /\langle\rho(E)\rangle]$, its distribution function acquires the log-normal form

$$
f(x) \approx \exp \left[-\left(x+\sigma^{2} / 2\right)^{2} /\left(2 \sigma^{2}\right)\right] / \sqrt{2 \pi \sigma^{2}},
$$

where $\sigma^{2}$ is given by Eq. (6).

Discussions. In Fig. 3 we compare the average DOS obtained from numerical solution of Hamiltonian (1) with the Dynes ansatz. We observe a reasonable agreement at weak disorder $W=0.5$ and not too small energies. We note that the Dynes parameter $\Gamma$ depends linearly on $1 / L^{2}$ (see the inset in Fig. 3). This behavior is caused by our choice of the number of Chebyshev polynomials $N_{\mathcal{C}}$ used in the expansion of the LDOS [51].

We continue the discussion with the LDOS variance. A detailed comparison between the numerical data at weak disorder $W=0.5$ and the analytical prediction Eq. (6) is presented in Fig. 4. The logarithmic growth of the LDOS variance 


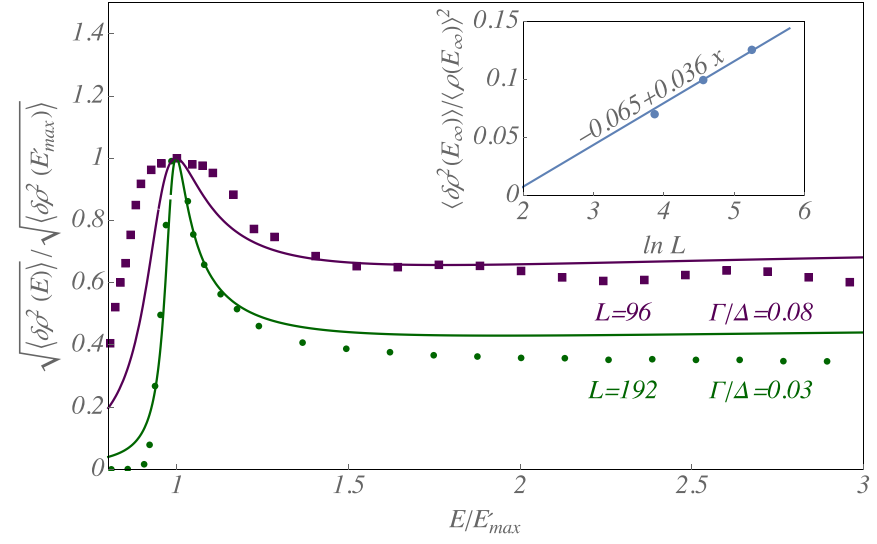

FIG. 4. The LDOS variance. Comparison between numerics and theory. The parameters are the same as in Fig. 3. Fitting to Eq. (6), we extract $g=35.4$ and $\ell=6$ for the fit. The position of the maximum is $\tilde{E}_{\max } \approx 0.97$ for $L=192$ and $\tilde{E}_{\max } \approx 1.03$ for $L=96$. The inset: The LDOS variance at large energies $E_{\infty} \simeq 3 \Delta$ versus system size for $L=48,96,192$.

with the system size agrees with numerical data as shown in the inset of Fig. 4. Incidentally, the logarithmic dependence of the LDOS variance on $L$ allows us to extract values of $g$ and $\ell$.

In agreement with numerical data, see Fig. 2, Eq. (6) predicts that the DOS variance has the maximum situated at energy $\tilde{E}_{\max }$ which is smaller than the energy $E_{\max }$ of the LDOS maximum. In particular, one can find $E_{\max }-\tilde{E}_{\max } \propto \Gamma / \ln \left(L_{\sqrt{\Gamma \Delta}} / \ell\right) \ll \Gamma$. We note that the difference $E_{\max }-\tilde{E}_{\max }$ is enlarged with increase in $\Gamma$, i.e., of disorder. In accordance with Eq. (6), the height of the maximum in $\left\langle[\delta \rho(E, r)]^{2}\right\rangle$ becomes on the order of $\left[4 \rho_{0}^{2} \Delta /(\pi g \Gamma)\right] \ln \left(L_{\sqrt{\Gamma \Delta}} / \ell\right)$. Again, this result is in agreement with the numerical data in Fig. 2 in which the height of the maximum of the DOS variance is enhanced with increasing disorder. Therefore, the expression (6) provides a reasonable description of the LDOS variance for a weak disorder.

In Fig. 5 we present a comparison of the distribution function for the logarithm of the normalized LDOS taken at two energies $E=E_{\max }$ and $E=3 E_{\max }$ and obtained from the numerical solution of Hamiltonian (1) against the theoretical prediction of weak multifractality theory Eq. (8). There is reasonable agreement between numerics and the theory. Also in Fig. 5 we plot the curves which corresponds to normal (rather log-normal) distribution of the LDOS (with the same variance). As one can see, the log-normal distribution is much more in agreement with numerical data for the distribution function than the normal distribution. This supports that the fluctuations of LDOS seen in numerics are of multifractal origin.

Finally, we mention that our numerical and analytical results are also in qualitative agreement with the experimental data [52]. Although the average and variance of the local DOS computed numerically as well as analytically demonstrate all features observed in the experiments, a more quantitative comparison is not indicated at this point; it would require

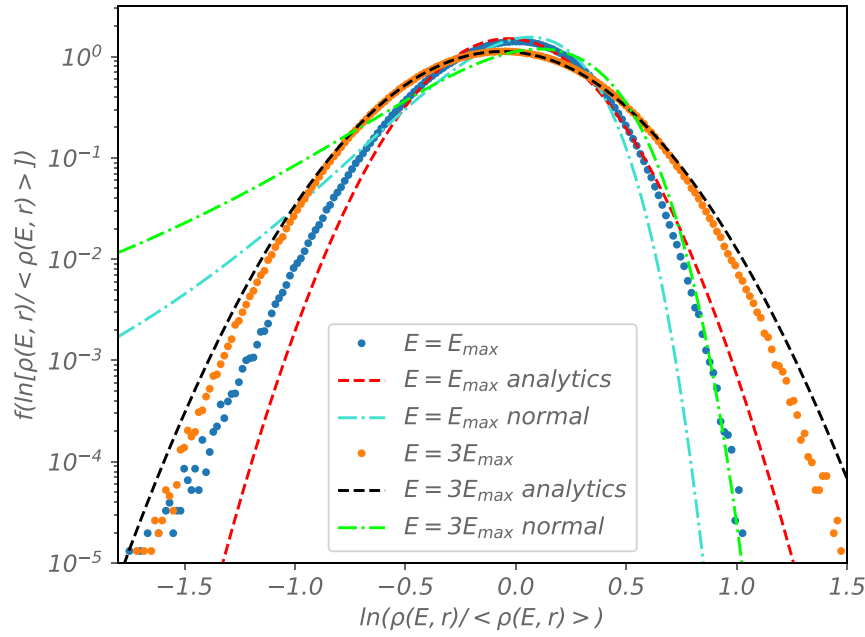

FIG. 5. The distribution of the logarithm of the normalized LDOS $x=\ln [\rho(E, \boldsymbol{r}) /\langle\rho(E)\rangle]$ at two different energies $E=E_{\max }$ and $E=3 E_{\max }$. The normal distribution for the LDOS in terms of the normalized LDOS has the following expression: $f_{\mathrm{n}}(x)=$ $\exp \left[x-\left(e^{x}-1\right)^{2} /\left(2 \sigma^{2}\right)\right] /\left(\sqrt{2 \pi \sigma^{2}}\right)$ with $\sigma^{2}=\left\langle\delta \rho^{2}(E, \boldsymbol{r})\right\rangle /\langle\rho(E)\rangle^{2}$ taken from numerics, see Fig. 4. (Parameters: $W=0.5 t$, $U=2.2 t, L=192$, and $N_{\mathcal{C}}=8192$.)

to include, e.g., also repulsive terms into our model, which goes beyond the scope of our present Letter. We note that in experimental samples the infrared logarithmic divergence of the variance should be cut off by the energy-dependent dephasing length (instead of the system size) [35].

Summary. To summarize we report the results of numerical and theoretical analyses of the energy dependence of fluctuations of the local DOS in weakly disordered superconducting films. We found that the local DOS has pronounced fluctuations and the variance has the energy dependence similar to the one for the average density of states. Our numerical and analytical approaches demonstrate that the DOS variance at energies higher than the energy gap diverging logarithmically with a system size $L$. Our numerical findings and the analytical results make up together coherent picture of multifractal correlations of the local DOS in weakly disordered superconducting films.

Acknowledgments. We thank M. Feigel'man, K. Franke, I. Gornyi, A. Mirlin, C. Strunk, I. Tamir, and W. Wulfhekel for discussions. We are especially grateful to I. Tamir and W. Wulfhekel for providing us the experimental data on the LDOS. The research was partially supported by the Russian Foundation for Basic Research (Grant No. 20-52-12013), Deutsche Forschungsgemeinschaft (Grants No. EV 30/11-1 and No. EV 30/14-1) cooperation, and by the Basic Research Program of HSE. The authors gratefully acknowledge the Gauss Centre for Supercomputing e.V. [53] for funding this project by providing computing time on the GCS Supercomputer SuperMUC at Leibniz Supercomputing Centre [54]. This work was performed on the supercomputer ForHLR funded by the Ministry of Science, Research and the Arts Baden-Wrttemberg and by the Federal Ministry of Education and Research. 
[1] A. A. Abrikosov and L. P. Gor'kov, On the theory of superconducting alloys, I. The electrodynamics of alloys at absolute zero, Sov. Phys. JETP 8, 1090 (1959).

[2] A. A. Abrikosov and L. P. Gor'kov, Superconducting alloys at finite temperatures, Sov. Phys. JETP 9, 220 (1959).

[3] P. W. Anderson, Theory of dirty superconductors, J. Phys. Chem. Solids 11, 26 (1959).

[4] L. N. Bulaevskii and M. V. Sadovskii, Localization and superconductivity, JETP Lett. 39, 640 (1984).

[5] M. Ma and P. A. Lee, Localized superconductors, Phys. Rev. B 32, 5658 (1985).

[6] A. Kapitulnik and G. Kotliar, Anderson Localization and the Theory of Dirty Superconductors, Phys. Rev. Lett. 54, 473 (1985).

[7] G. Kotliar and A. Kapitulnik, Anderson localization and the theory of dirty superconductors. II, Phys. Rev. B 33, 3146 (1986).

[8] D. B. Haviland, Y. Liu, and A. M. Goldman, Onset of Superconductivity in the Two-Dimensional Limit, Phys. Rev. Lett. 62, 2180 (1989).

[9] A. M. Goldman and N. Marković, Superconductor- insulator transitions in the two-dimensional limit, Phys. Today 51(11), 39 (1998).

[10] V. F. Gantmakher and V. T. Dolgopolov, Superconductorinsulator quantum phase transition, Phys.-Usp. 53, 1 (2010).

[11] B. Sacépé, M. Feigel'man, T. M. Klapwijk, Quantum breakdown of superconductivity in low-dimensional materials, Nat. Phys. 16, 734 (2020).

[12] S. Maekawa and H. Fukuyama, Localization effects in twodimensional superconductors, J. Phys. Soc. Jpn. 51, 1380 (1982).

[13] H. Takagi and Y. Kuroda, Anderson localization and superconducting transition temperature in two-dimensional systems, Solid State Commun. 41, 643 (1982).

[14] S. Maekawa, H. Ebisawa, and H. Fukuyama, Theory of dirty superconductors in weakly localized regime, J. Phys. Soc. Jpn. 53, 2681 (1984).

[15] P. W. Anderson, K. A. Muttalib, and T. V. Ramakrishnan, Theory of the "universal" degradation of $T_{c}$ in high-temperature superconductors, Phys. Rev. B 28, 117 (1983).

[16] C. Castellani, C. Di Castro, G. Forgacs, and S. Sorella, Spin orbit coupling in disordered interacting electron gas, Solid Stat. Commun. 52, 261 (1984).

[17] L. N. Bulaevskii and M. V. Sadovskii, Anderson localization and superconductivity, J. Low Temp. Phys. 59, 89 (1985).

[18] A. M. Finkel'stein, Superconducting transition temperature in amorphous films, JETP Lett. 45, 46 (1987).

[19] T. R. Kirkpatrick and D. Belitz, Logarithmic Corrections to Scaling Near the Metal-Insulator Transition, Phys. Rev. Lett. 70, 974 (1993).

[20] T. R. Kirkpatrick and D. Belitz, Cooperons at the metalinsulator transition revisited: Constraints on the renormalization group and a conjecture, Phys. Rev. B 50, 8272 (1994).

[21] A. M. Finkel'stein, Suppression of superconductivity in homogeneously disordered systems, Physica B 197, 636 (1994).

[22] M. V. Feigel'man, L. B. Ioffe, V. E. Kravtsov, and E. A. Yuzbashyan, Eigenfunction Fractality and Pseudogap State Near the Superconductor-Insulator Transition, Phys. Rev. Lett. 98, 027001 (2007).
[23] M. V. Feigel'man, L. B. Ioffe, V. E. Kravtsov, and E. Cuevas, Fractal superconductivity near localization threshold, Ann. Phys. (NY) 325, 1390 (2010).

[24] I. S. Burmistrov, I. V. Gornyi, and A. D. Mirlin, Enhancement of the Critical Temperature of Superconductors by Anderson Localization, Phys. Rev. Lett. 108, 017002 (2012).

[25] I. S. Burmistrov, I. V. Gornyi, and A. D. Mirlin, Superconductor-insulator transitions: Phase diagram and magnetoresistance, Phys. Rev. B 92, 014506 (2015).

[26] F. Evers and A. D. Mirlin, Anderson transitions, Rev. Mod. Phys. 80, 1355 (2008).

[27] M. N. Gastiasoro and B. M. Andersen, Enhancing superconductivity by disorder, Phys. Rev. B 98, 184510 (2018).

[28] B. Fan and A. M. García-García, Superconductivity at the threedimensional Anderson metal-insulator transition, Phys. Rev. B 102, 184507 (2020).

[29] K. Zhao, H. Lin, X. Xiao, W. Huang, W. Yao, M. Yan, Y. Xing, Q. Zhang, Z.-X. Li, S. Hoshino, J. Wang, S. Zhou, L. Gu, M. S. Bahramy, H. Yao, N. Nagaosa, Q.-K. Xue, K. T. Law, X. Chen, and S.-H. Ji, Disorder-induced multifractal superconductivity in monolayer niobium dichalcogenides, Nat. Phys. 15, 904 (2019).

[30] C. Rubio-Verdú, A. M. García-García, H. Ryu, D.-J. Choi, J. Zaldívar, S. Tang, B. Fan, Z.-X. Shen, S.-K. Mo, J. I. Pascual, and M. M. Ugeda, Visualization of multifractal superconductivity in a two-dimensional transition metal dichalcogenide in the weak-disorder regime, Nano Lett. 20, 5111 (2020).

[31] J. Mayoh and A. M. García-García, Global critical temperature in inhomogeneous superconductors induced by multifractality, Phys. Rev. B 92, 174526 (2015).

[32] I. S. Burmistrov, I. V. Gornyi, and A. D. Mirlin, Local density of states and its mesoscopic fluctuations near the transition to a superconducting state in disordered systems, Phys. Rev. B 93, 205432 (2016).

[33] B. Fan and A. M. García-García, Enhanced, phase coherent, multifractal-like, two-dimensional superconductivity, Phys. Rev. B 101, 104509 (2020).

[34] M. Stosiek, B. Lang, and F. Evers, Self-consistent-field ensembles of disordered Hamiltonians: Efficient solver and application to superconducting films, Phys. Rev. B 101, 144503 (2020).

[35] I. S. Burmistrov, I. V. Gornyi, and A. D. Mirlin, Multifractallyenhanced superconductivity in thin films, Ann. Phys. (N.Y.) (2021), doi:10.1016/j.aop.2021.168499.

[36] B. Sacépé, C. Chapelier, T. I. Baturina, V. M. Vinokur, M. R. Baklanov, and M. Sanquer, Disorder-Induced Inhomogeneities of the Superconducting State Close to the SuperconductorInsulator Transition, Phys. Rev. Lett. 101, 157006 (2008).

[37] B. Sacépé, T. Dubouchet, C. Chapelier, M. Sanquer, M. Ovadia, D. Shahar, M. Feigel'man, and L. Ioffe, Localization of preformed Cooper pairs in disordered superconductors, Nat. Phys. 7, 239 (2011).

[38] M. Chand, G. Saraswat, A. Kamlapure, M. Mondal, S. Kumar, J. Jesudasan, V. Bagwe, L. Benfatto, V. Tripathi, and P. Raychaudhuri, Phase diagram of the strongly disordered s-wave superconductor $\mathrm{NbN}$ close to the metal-insulator transition, Phys. Rev. B 85, 014508 (2012).

[39] G. Lemarié, A. Kamlapure, D. Bucheli, L. Benfatto, J. Lorenzana, G. Seibold, S. C. Ganguli, P. Raychaudhuri, and C. Castellani, Universal scaling of the order-parameter distri- 
bution in strongly disordered superconductors, Phys. Rev. B 87, 184509 (2013).

[40] Y. Noat, V. Cherkez, C. Brun, T. Cren, C. Carbillet, F. Debontridder, K. Ilin, M. Siegel, A. Semenov, H.-W. Hübers, and D. Roditchev, Unconventional superconductivity in ultrathin superconducting $\mathrm{NbN}$ films studied by scanning tunneling spectroscopy, Phys. Rev. B 88, 014503 (2013).

[41] C. Carbillet, S. Caprara, M. Grilli, C. Brun, T. Cren, F. Debontridder, B. Vignolle, W. Tabis, D. Demaille, L. Largeau, K. Ilin, M. Siegel, D. Roditchev, and B. Leridon, Confinement of superconducting fluctuations due to emergent electronic inhomogeneities, Phys. Rev. B 93, 144509 (2016).

[42] C. Carbillet, V. Cherkez, M. A. Skvortsov, M. V. Feigel'man, F. Debontridder, L. B. Ioffe, V. S. Stolyarov, K. Ilin, M. Siegel, D. Roditchev, T. Cren, and C. Brun, Spectroscopic evidence for strong correlations between local superconducting gap and local Altshuler-Aronov density of states suppression in ultrathin $\mathrm{NbN}$ films, Phys. Rev. B 102, 024504 (2020).

[43] J. Hubbard, Electron correlations in narrow energy bands, Proc. R. Soc. London, Ser. A 276, 238 (1963).

[44] Within the self-consistency cycles $\Delta\left(\boldsymbol{r}_{i}\right)$ becomes essentially a random quantity but with highly nontrivial correlations contrary to "ad hoc" models with uncorrelated random pairing amplitudes, see e.g. Ref. [45].
[45] D. E. Katsanos, S. N. Evangelou, and C. J. Lambert, Superconductivity-induced Anderson localization, Phys. Rev. B 58, 2442 (1998).

[46] A. Weiße, G. Wellein, A. Alvermann, and H. Fehske, The kernel polynomial method, Rev. Mod. Phys. 78, 275 (2006).

[47] A. D. Mirlin, Statistics of energy levels and eigenfunctions in disordered systems, Phys. Rep. 326, 259 (2000).

[48] R. C. Dynes, V. Narayanamurti, and J. P. Garno, Direct Measurement of Quasiparticle-Lifetime Broadening in a StrongCoupled Superconductor, Phys. Rev. Lett. 41, 1509 (1978).

[49] P. A. Lee and T. V. Ramakrishnan, Disordered electronic systems, Rev. Mod. Phys. 57, 287 (1985)

[50] I. V. Lerner, Distribution functions of current density and local density of states in disordered quantum conductors, Phys. Lett. A 133, 253 (1988).

[51] We choose $N_{\mathcal{C}}$ such that the number of states within the resulting energetic broadening is constant for all system sizes. Thus the $1 / L^{2}$ behavior is a reflection of the dependence of the mean level spacing on system size. Although there is an intrinsic broadening caused by disorder, it is negligible with respect to the broadening brought about by our numerical procedure.

[52] I. Tamir and W. Wulfhekel (private communication).

[53] http://www.gauss-centre.eu.

[54] http://www.lrz.de. 